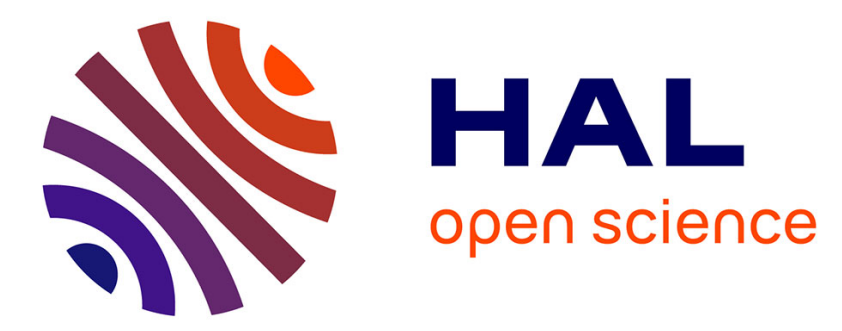

\title{
Shearing of a confined granular layer : Tangential stress and dilatancy
}

Christophe Coste

\section{To cite this version:}

Christophe Coste. Shearing of a confined granular layer: Tangential stress and dilatancy. Physical Review E: Statistical, Nonlinear, and Soft Matter Physics, 2004, 70 (5), pp.70-81. 10.1103/PhysRevE.70.051302 . hal-01406731

\section{HAL Id: hal-01406731 \\ https://hal-univ-paris.archives-ouvertes.fr/hal-01406731}

Submitted on 1 Dec 2016

HAL is a multi-disciplinary open access archive for the deposit and dissemination of scientific research documents, whether they are published or not. The documents may come from teaching and research institutions in France or abroad, or from public or private research centers.
L'archive ouverte pluridisciplinaire HAL, est destinée au dépôt et à la diffusion de documents scientifiques de niveau recherche, publiés ou non, émanant des établissements d'enseignement et de recherche français ou étrangers, des laboratoires publics ou privés.

\section{(c)(1)}

Distributed under a Creative Commons Attribution| 4.0 International License 


\title{
Shearing of a confined granular layer: Tangential stress and dilatancy
}

\author{
C. Coste \\ Groupe de Physique des Solides, Campus Boucicaut 140 rue de Lourmel, 75015 Paris, France
}

(Received 11 June 2004; published 5 November 2004)

\begin{abstract}
We study the behavior of a confined granular layer under shearing, in an annular cell, at low velocity. We give evidence that the response of the granular layer under shearing is described by characteristic length scales. The tangential stress reaches its steady state on the same length scale as the dilatancy. Stop-and-go experiments performed at several driving velocities show a logarithmic increase of the static friction coefficient with waiting time, followed by rejuvenation on a characteristic length of the order of the magnitude of a Hertz contact between adjacent grains. The dilatancy does not evolve during the stop, neither during the elastic reloading when the driving is resumed. There is a small variation when sliding sets anew, which corresponds to the rejuvenation of the layer, and this variation is independent of the waiting time. We argue that aging is due to the behavior of individual contacts between grains, not global evolution of the piling. Under an instantaneous increase of the velocity, the tangential stress reaches a new steady state, exhibiting velocity strengthening behavior. An increase of dilatancy is also observed. It is much larger than fluctuations in the steady state, variations in a stop and-go-experiment, but much less than for shearing of freshly poured grains. The dilatancy variation during a velocity jump is not due to structural rearrangements of the piling. The evolutions of tangential stress and dilatancy are logarithmic in the ratio of upper and lower velocities.
\end{abstract}

DOI: $10.1103 /$ PhysRevE.70.051302

PACS number(s): $45.70 . \mathrm{Mg}$

\section{INTRODUCTION}

The behavior of a sheared granular medium is important for the design of silos and hoppers used to handle and store many industrial materials [1] or for the understanding of the frictional behavior of earthquake faults [2,3]. This is also a fundamental question of granular mechanics. In this paper, we are concerned with low shear rates [see below, Eq. (1)]. Problems of interest in this regime include stick-slip instabilities [4,5], coupling between friction and dilatancy $[4,6-8]$, behavior of the granular layer at large shearing distances $[9,10]$, shear banding and strain localization $[3,6,10-13]$, aging $[5,12,14,15]$, and response to an instantaneous velocity jump $[3,7,16]$. In what follows, we will insist on the approach toward a steady state, then discuss aging under constant normal and tangential stress, and last the response to velocity jumps.

Many laboratory devices may be used to study the response of a granular layer to shear. The simplest apparatus may be a plate, placed on the top of a granular layer, driven at a controlled velocity $[8,14]$. The granular matter may be put into two boxes, separated by a small gap, in relative translation and under normal stress: the direct shear box [6]. Geophysicists simulate the granular matter contained in faults by inserting a granular layer (the gouge) between two granite blocks; the relative translation and normal stress are applied to those blocks [7,15]. An inconvenience of those setups is that the displacement is limited to a few centimeters. One way to avoid this limitation is to use a Couette geometry $[10,12,13]$. In this geometry, experiments have been done at constant density $[12,13]$ or under constant pressure by submitting the granular layer to a radial stress [10]. A third possibility is the use of an annular plane Couette geometry $[3-5,9,16]$, in which the grains are contained in an annulus, relative motion imposed between this annulus and the cover plate, and constant pressure applied on the cover plate.
If the mean radius of the annulus is large compared to its width, we can neglect the radial velocity gradient and this setup is very similar to the direct shear box. This is the geometry used in the present study.

A peculiarity of our shearing apparatus is that the grains cannot escape from the cell, allowing unambiguous measurements of the layer dilatancy. When poured in the experimental cell, the grains are in a dense state. The average volume fraction is $0.61 \% \pm 1 \%$ (see below, Sec. II B), very close to the so called random close packing of 0.637 [17]. In this regime, shearing necessarily implies dilatancy of the granular layer [18]. The coupling between shear stress and dilatancy is still an open problem in granular media mechanics, about which we provide experimental information.

Our experiments are done at low driving velocity $V$, typically $V \in\left[3 \times 10^{-3}, 1\right] d / \mathrm{s}$ in units of grain diameter $d$ per second. More precisely, we may define a dimensionless shear rate $I[19]$, which compares the relative importance of inertia and confining stresses. With the $\Omega$ angular velocity, $\bar{R}$ the mean cell radius, $H$ the cell depth, $\rho$ the grain density, and $N$ the normal stress, we set

$$
I \equiv \frac{(\Omega \bar{R} / H) d}{\sqrt{N / \rho}} \in\left[2 \times 10^{-6}, 2 \times 10^{-4}\right] .
$$

We are thus always in a very-low-shear-rate regime.

In our experiments, we impose the driving velocity and normal stress to the system and measure global quantities, the average tangential stress, and the average dilatancy of the layer. We carefully compare the dynamics of those two quantities. We study the transient to reach a steady shearing, beginning with freshly poured grains, under a constant driving velocity. We then discuss stop-and-go experiments and aging and the system response to instantaneous velocity jumps. 


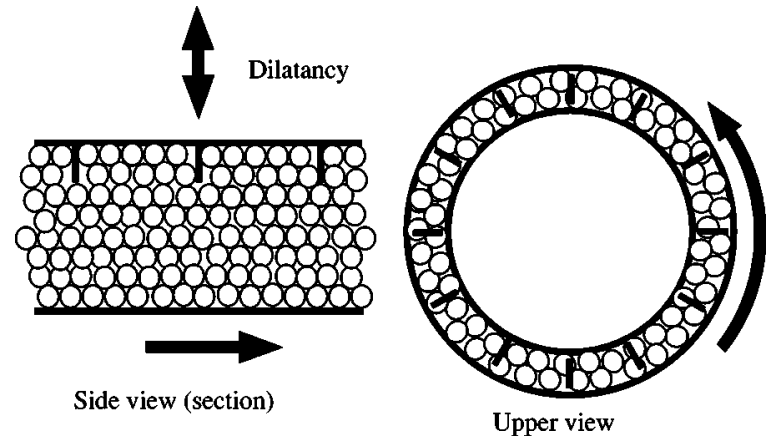

FIG. 1. Sketch of the apparatus. The drawing on the right is an upper view, showing the cell that contains the beads and the 12 steel teeth of the cover that are buried in them. The large arrow indicates the imposed rotation of the cell; the cover is blocked by a cantilever spring (not shown in the drawing), the deflection of which gives the tangential stress applied on the beads. The drawing on the left is a cutting of the side view. The bottom arrow indicates the imposed motion of the cell, while the top arrow indicates the motion of the cover, moving freely in the vertical direction because of the dilatancy of the granular layer.

In Sec. II, we describe our experimental configuration. Section III is devoted to the presentation and discussion of our experimental results. More specifically, we discuss steady-state shearing of freshly poured grains in Sec. III A, stop-and-go shear experiments in Sec. III B, and velocity jumps in Sec. III C. The conclusions are summarized in Sec. IV.

\section{EXPERIMENTAL SETUP}

\section{A. Description of the apparatus}

The experimental setup is an annular cell of inner radius $103 \mathrm{~mm}$, outer radius $117 \mathrm{~mm}$, and depth $20 \mathrm{~mm}$. It is placed on an annular rotation stage (Newport RV240PP), with a stepper motor drive. The cover plate is prevented from following the rotation by using a cantilever spring, of stiffness $k=2.25 \times 10^{5} \mathrm{~N} / \mathrm{m}$. In order to shear the bulk of the granular layer, the cover plate has steel teeth, $5 \mathrm{~mm}$ long, every $30^{\circ}$ (see Fig. 1). With this setup, shearing occurs in the plane tangent to the teeth extremities.

The cell width $(14 \mathrm{~mm})$ is much smaller than its mean radius $\bar{R}(110 \mathrm{~mm})$, so that in the following we consider the shearing velocity $V$ as almost uniform in width, simply related to the angular velocity $\Omega$ by $V=\bar{R} \Omega$. This has been shown to be basically correct for annular cells much larger in comparison with their mean radius [5]. The angular velocity $\Omega$ ranges from $50 \times 10^{-6}$ to $16 \times 10^{-3} \mathrm{rad} / \mathrm{s}$, so that the velocity ranges from $5 \mu \mathrm{m} / \mathrm{s}$ to $1750 \mu \mathrm{m} / \mathrm{s}$, that is, from 3 $\times 10^{-3} d / \mathrm{s}$ to $1 \mathrm{~d} / \mathrm{s}$ in units of a grain diameter $d$ $\approx 1.5 \mathrm{~mm}$. The experiments are thus all done in the lowshear-rate regime $I \ll 1$ [see Eq. (1)].

The cover plate is free to move vertically, and three position sensors measure its height. With those three measurement points, we get the mean displacement of the cover, hence the dilatancy of the granular layer. The cell and cover are designed in such a way that no grain can escape during the shearing, despite a gap between the cover and annulus that prevents friction between them.

The deflection of the cantilever spring is measured by another position sensor, in order to know the mean shearing stress applied to the granular layer. Some masses may be added to the cover plate, so that the weight is in the range $[32,110] N$ (which corresponds to a normal stress $N$ between 3.30 and $11.34 \mathrm{kPa}$ ). The weight of the cover alone is more than 12 times that of the grains themselves.

\section{B. Preparation of a sample}

The grains are glass beads, of diameter $1.5 \% \pm 10 \% \mathrm{~mm}$. Before using them in an experiment, we wash them carefully in distilled water with an ultrasonic cleaner. This manipulation spectacularly decreases the wear during the experiments: it allows up to $15 \mathrm{~m}$ of cumulated sheared distance without any systematic variation on the effective friction coefficient or dilatancy. It suppresses also stick-slip oscillations during shearing.

In order to improve the reproducibility of our experiments, we prepare all samples in the same way. The grains are poured with the help of a hopper, maintaining a constant angular velocity of the empty cell of $20^{\circ} / \mathrm{s}$. When the cell is full, the upper surface of the grain is not flat. We thus change the velocity down to $5^{\circ} / \mathrm{s}$ and drop the beads that may be in excess with the help of a rake. At the end of this process, the height of the grains layer is $20 \mathrm{~mm} \pm 75 \mu \mathrm{m}$, and its mass is $266 \pm 2 \mathrm{~g}$-, that is, an average volume fraction of $0.61 \% \pm 1 \%$. Granular media dynamics is oversensitive to small variations in density [20], and this uncertainty is most likely responsible for the scatter of the effective dynamical friction coefficient (see below section III A).

The zero of dilatancy is, in all experiments, chosen to be the position of the cell cover when we place it on the freshly poured grains. Ideally, the zero should be fixed once and for all. There are nevertheless two causes of inaccuracy. The first is that we have to remove the sensors to pull up the cell cover and empty the cell, and we cannot be sure to replace them exactly in the same fashion from one experimental run to the other. The second is that despite our care, the upper surface of the grains is not perfectly flat and not at the same mean position after each pouring process. The zero is thus known to a precision of $\pm 75 \mu \mathrm{m}$. This value is used above to estimate the uncertainty on the depth of the granular layer.

\section{EXPERIMENTAL RESULTS}

\section{A. Shearing of freshly poured grains}

In Fig. 2, we display the evolution of the tangential stress $T$, normalized by the normal stress $N$ (upper plot), and of the dilatancy as a function of the displacement $L$ (lower plot). The rotation angle $\theta$ is defined as the product of the driving angular velocity by the time since we start the motor. During elastic loading of the cantilever spring, $\theta$ does not represent a shearing angle, but rather the deformation of the spring. In what follows we are interested in much larger rotations. The linear displacement $L$ is defined as $L \equiv \bar{R} \theta$. The experiments shown in Fig. 2 are done for a normal stress of $9.73 \mathrm{kPa}$ and 

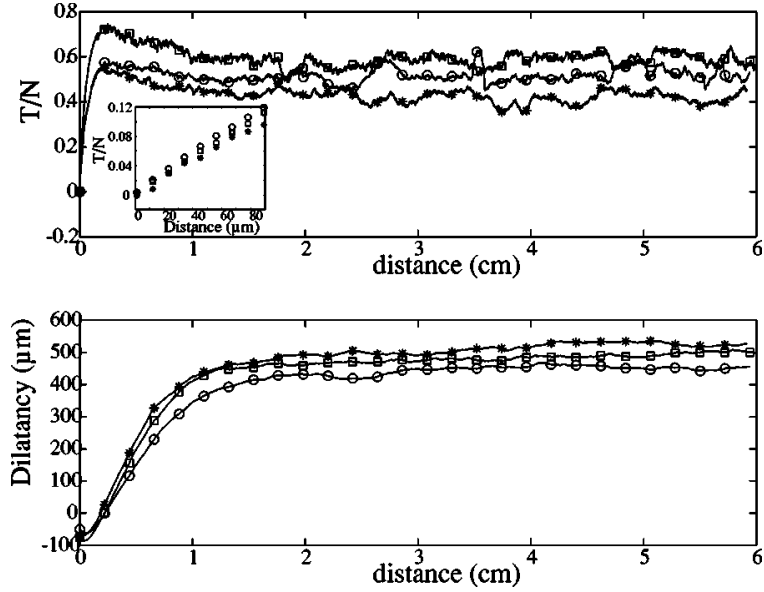

FIG. 2. Plot of $T / N$ (upper curves) and the dilatancy (in $\mu \mathrm{m}$, lower curves) as a function of displacement (in $\mathrm{cm}$ ), for the same normal stress $(9.73 \mathrm{kPa})$ and several driving velocities: $11 \mu \mathrm{m} / \mathrm{s}$ (○), $110 \mu \mathrm{m} / \mathrm{s}(\square)$, and $1100 \mu \mathrm{m} / \mathrm{s}$ (*). In the upper curve, the inset shows the very begining of the motion, when elastic response of the layer occurs. The data correspond to three different initial states of freshly poured grains. The spatial resolution (1 point per micrometer) is the same for all data, but for clarity the symbols are shown each 1000 points only. The properties of the curves are summarized in Table I.

driving velocities respectively equal to 11,110 , and $1100 \mu \mathrm{m} / \mathrm{s}$.

Let us first consider the evolution of the tangential stress. The initial response of the granular layer to the driving, along the first $90 \mu \mathrm{m}$, is shown in the inset. We see that the tangential stress evolves linearly with distance. Moreover, this evolution is reversible and no hysteresis is observed when we reverse the direction of rotation. This initial response is thus elastic, with a effective stiffness of 2.8 $\times 10^{5} \mathrm{~N} / \mathrm{m}$, which does not depend on the velocity. Beyond typically $150 \mu \mathrm{m}$, the response is plastic and hysteresis becomes observable. If we continue further the motion of the annular cell, the tangential stress reaches a maximum $T$ $=\mu_{s} N$, then decreases toward a stationary value $T=\mu_{d} N$ (apart from fluctuations, which are dealt with below). The maximum $\mu_{s}$ may be defined as a static friction coefficient. When it has been reached, the granular layer undergoes sliding at a constant velocity $V$. The coefficient $\mu_{d}$, defined as the average of $T / N$ in this steady state, may indeed be identified with a dynamic friction coefficient $\mu_{d}<\mu_{s}$, as for solid friction. When we plot the tangential stress in the steady state as a function of the normal stress, as in Fig. 3, we indeed obtain a straight line, with a slope $\mu_{d} \approx 0.54$.

In the experiments shown in Fig. 2, an additional mass of $6.33 \mathrm{~kg}$ is placed on the cover, which explains why the initial dilatancy is negative. The evolution of dilatancy, shown in the lower curve, is typical of dense granular pilings ([1], Sec. 6.5). There is first compaction (about $50 \mu \mathrm{m}$ ), then dilatancy of the layer up to $450 \mu \mathrm{m}$. The order of magnitude of this effect may be recovered in a very simple fashion. As a very crude approximation, suppose an initial regular HCP lattice. A bead is supported by three others, and its center is at a height $R \sqrt{8 / 3}$ from the plane defined by the three bead cen-

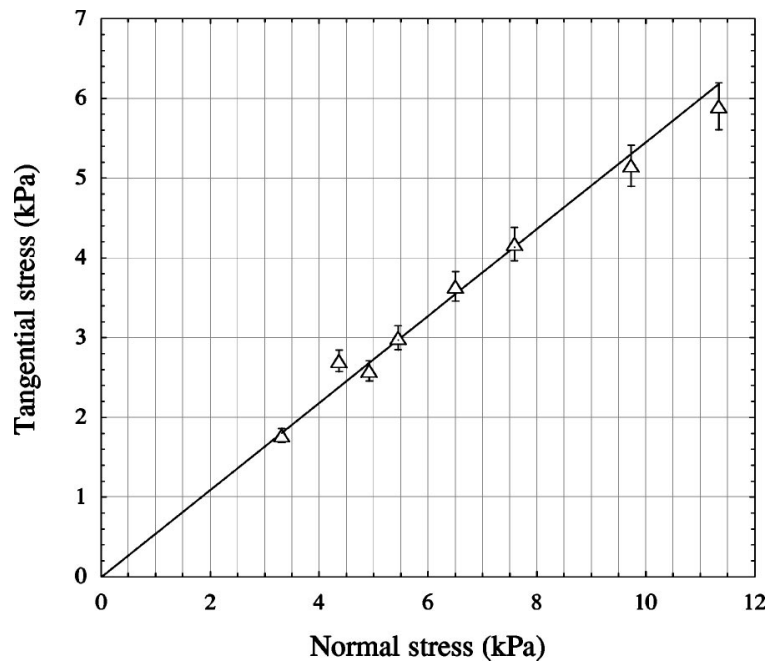

FIG. 3. Plot of the tangential stress (in $\mathrm{kPa}$ ) as a function of the normal stress (in $\mathrm{kPa}$ ). The tangential stress is measured in the steady state, for a driving velocity of $11 \mu \mathrm{m} / \mathrm{s}$. The error bars correspond to the $7 \%$ fluctuations level observed in the steady state. The slope of the straight line, which is constrained to go through the origin, is the average of all values of $\mu_{d},\left\langle\mu_{d}\right\rangle=0.54$.

ters. The bead may slide if it increases its height up to $R \sqrt{3}$, being in contact with only two of the underlying beads. Hence the dilatancy is $\Delta h \approx N R(\sqrt{3}-\sqrt{8 / 3})$, where $N \approx 10$ is the number of beads between the teeth extremities and the cell bottom. This gives $\Delta h \approx 750 \mu \mathrm{m}$, which is an overestimate since the initial experimental packing fraction of 0.61 is less than the HCP one of 0.74 .

The striking feature of Fig. 2 is that either the tangential stress (upper curves) or the dilatancy (lower curves) evolves in the same fashion with distance, rather independently from the velocity, which varies by two orders of magnitude. Those evolutions are characterized by typical length scales, not by time scales.

This discussion may be made more precise if we define several typical length scales for the system. First we introduce $L\left(\mu_{s}\right)$, which is the distance necessary to reach the maximum of tangential stress, after the elastic loading of the granular layer. Then we may define $L\left(\mu_{d}\right)$, as the distance necessary to reach the tangential stress steady state. In order to get a result which does not depend too much on fluctuations, we plot the tangent to the curve $T / N$, shortly after the maximum. We then define $L\left(\mu_{d}\right)$ as the necessary distance to decrease toward $\mu_{d}$ along this tangent.

Let us now consider the evolution of the dilatancy. We define a length scale $L\left(h_{\min }\right)$ as the position of the minimum of dilatancy. Then we introduce $L\left(h_{\infty}\right)$ as the distance necessary to reach the steady-state dilatancy $h_{\infty}$. We take advantage of the fact that two straight lines naturally appear, tangent to the experimental curve: one during the almost linear increase of dilatancy that follows the minimum and the other almost parallel to the abcissa axis in the stationary regime. The intersection of those two straight lines provides a good definition of $L\left(h_{\infty}\right)$, quite insensitive to fluctuations.

The values of those four different length scales are listed in Table I, which shows that indeed they do not depend on 
TABLE I. Measurements corresponding to Fig. 2. $V$ is the driving velocity, $\mu_{s}$ the static friction coefficient, $L\left(\mu_{s}\right)$ the length at which it is attained, $\mu_{d}$ the steady-state friction coefficient, and $h_{\infty}$ the steady-state dilatancy. The lengths $L\left(\mu_{d}\right)$ and $L\left(h_{\infty}\right)$ are the distances of shearing necessary to reach those steady states (see text for their precise definition), and finally $L\left(h_{\min }\right)$ is the position of the dilatancy minimum.

\begin{tabular}{lccccccc}
\hline \hline$V(\mu \mathrm{m} / \mathrm{s})$ & $\mu_{s}$ & $L\left(\mu_{s}\right)(\mathrm{mm})$ & $\mu_{d}$ & $L\left(\mu_{d}\right)(\mathrm{mm})$ & $h_{\infty}(\mu \mathrm{m})$ & $L\left(h_{\infty}\right)(\mathrm{mm})$ & $L\left(h_{\min }\right)(\mathrm{mm})$ \\
\hline 11 & 0.65 & 2.2 & $0.52 \pm 0.03$ & 8 & $453 \pm 5$ & 11 & 0.6 \\
110 & 0.75 & 2.5 & $0.59 \pm 0.03$ & 9.5 & $488 \pm 4$ & 9 & 0.5 \\
440 & 0.75 & 3 & $0.60 \pm 0.04$ & 8.5 & $413 \pm 4$ & 10 & 0.8 \\
1100 & 0.6 & 2.5 & $0.53 \pm 0.03$ & 10 & $520 \pm 5$ & 9 & 0.5 \\
1760 & 0.77 & 2.2 & $0.67 \pm 0.04$ & 7.5 & $440 \pm 6$ & 10.5 & 0.5 \\
\hline \hline
\end{tabular}

the driving velocity. This result is quite obvious for $L\left(\mu_{s}\right)$. It only evidences that there is indeed a static friction coefficient, which implies a sufficient deformation of the cantilever spring in order for sliding to begin. This length scale thus appears because of the measuring process. This explanation is not relevant for the other length scales. Their very existence is strong evidence that the collective behavior of the layer is characterized by length scales, not time scales.

The data collected in Table I show that, at all velocities, $L\left(h_{\infty}\right) \approx L\left(\mu_{d}\right)$. The stationary state for the dilatancy corresponds to the one for the tangential stress. Recent theoretical approaches [21,22] insist on the coupling between shear stress and dilatancy (or free volume). Our data show that indeed the respective evolutions of the dilatancy and tangential stress occur on the same length scales. The order of magnitude of this length scale is 7 bead diameters in our experiment. In the experiment of Chambon et al. [10], for a Couette geometry, the typical displacement to reach the steady state is of the order of 10 grain diameters (for a velocity of $83 \mu \mathrm{m} / \mathrm{s}$, a radial stress of $500 \mathrm{kPa}$, and a gap between cylinders of 100 grain diameters). In the experiments of Shibuya et al. [6], two boxes of depth 750 grain diameters slide one on the other (for a velocity of $4 \mu \mathrm{m} / \mathrm{s}$ and a normal stress of $50 \mathrm{kPa}$ ). If we consider Fig. 12 of their paper, we see that the displacement is sufficient to reach the steady state and evaluate $L\left(h_{\infty}\right) \approx L\left(\mu_{d}\right) \approx 4 \mathrm{~mm}$ - that is, 25 grain diameters. Those values are in reasonable agreement and show that $L\left(h_{\infty}\right)$ and $L\left(\mu_{d}\right)$ scale with the grain diameter, not the depth of the layer.

In the experiment of Géminard et al. [8], the grains are immersed in water and the normal stress very small (20 Pa), so it is difficult to make a direct comparison. Nevertheless, their observations are quite the same as ours. They do observe that the steady state is reached for both tangential stress and dilatancy after a displacement of typically 5 grain diameters, for a depth of 30 particles (see their Fig. 2).

In granular media, the deformation is localized in shear bands, first observed in triaxial experiments [23,24], typically extending to 10 grain diameters. Shear bands have been observed in the experiments of Chambon et al. [10], with a 7 grain width, and in the experiments of Shibuya et al. [6], with a 20 grain width. In our experiment, the deformation presumably extends from the extremities of the steel teeth toward the bottom of the cell-that is, 10 grains. Thus, in each case, the length scale necessary to reach the steady state is the same as the width of the shear band. This is confirmed by direct observation of the spatial distribution of stresses, using photoelastic grains in a two dimensional (2D) Couette geometry [9]. In the steady state the orientation of the force chains in the shear band is indeed roughly $45^{\circ}$ to the direction of shear. Our experiment shows that the stationary dilatancy is reached on a length scale of the same order of magnitude. It means that, in order to build a shear band, shearing must be performed along a distance equal to its width.

Let us add two remarks. The first one is that it is somewhat excessive to speak of shear bands in our setup, because there are typically ten glass beads between the cell bottom and the steel teeth, and thus the deformation presumably extends in the entire space between the teeth extremities and the bottom. The second is that this characteristic size of shear bands is strongly related to the fact that, in all the experiments discussed until now $[4-7,9,10,12,13,15,16]$, the shearing takes place at a solid boundary. Recent experiments, using a different setup where shear zones are created far from the sidewalls, show that no characteristic scale is observed for the shear zone [11].

Up to now, the focus has been on the mean values for tangential stress and dilatancy. As seen in Fig. 2, both quantities exhibit fluctuations, which may be characterized by the standard deviation of the data, in the steady state. Those of dilatancy are very small, less than $\pm 5 \mu \mathrm{m}$ or $1 \%$. It means that in steady-state sliding, rearrangements of the grains are made at almost constant density. The fluctuations of tangential stress are much greater, their amplitude $\Delta(T / N)$ being typically 0.035 - that is, $7 \%$ of $\mu_{d}$.

If we Fourier transform the tangential stress as a function of distance, in the steady state, the Fourier spectrum exhibits a peak at a small wave vector, as shown by the inset of Fig. 4. Taking the inverse of this wave vector, we may define a length scale $L_{\text {fluc }}$, characteristic of the fluctuations. We now want to see if this length scale depends on the driving velocity.

For the sake of comparison, the data were taken on a unique sample - that is, a unique initial density. We sheared this sample at several velocities, along a distance sufficient to collect enough data in the steady state. In practice, a 6$\mathrm{cm}$ displacement was done at each velocity, and we used the last $3 \mathrm{~cm}$ of the registered data for each run. With this method, the driving velocity is the only parameter that varies from one experimental point to the other.

In Fig. 4 we plot the amplitude of the tangential stress fluctuations, together with their characteristic length $L_{\mathrm{fluc}}$, as 


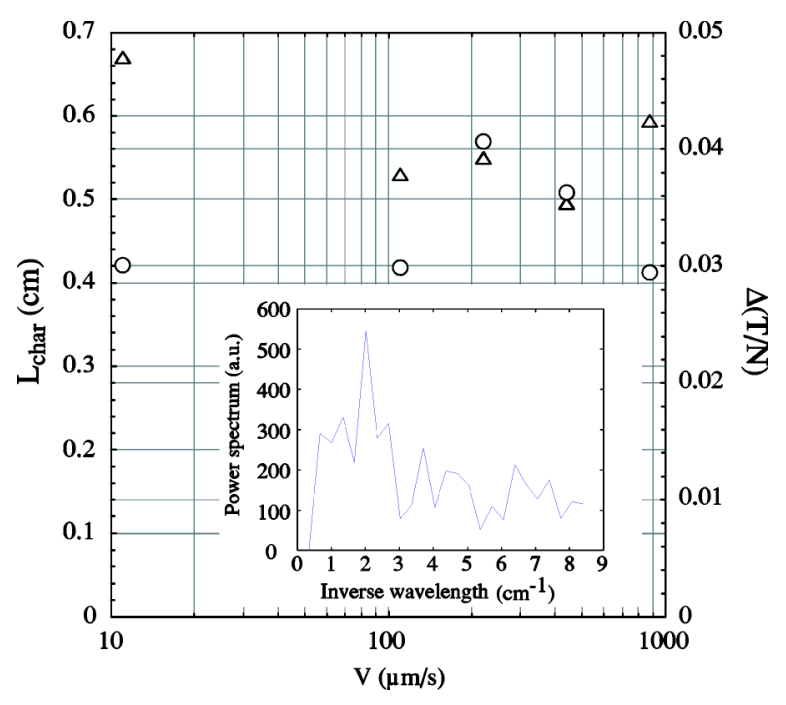

FIG. 4. Left ordinate, $\triangle$ : characteristic length $L_{\text {fluc }}$ of the fluctuations of normalized tangential stress (in $\mathrm{cm}$ ), in the steady state, as a function of the driving velocity (in $\mu \mathrm{m} / \mathrm{s}$ ). Right ordinate, $\bigcirc$ : amplitude $\Delta(T / N)$ of those fluctuations as a function of the driving velocity. The inset shows the power spectrum of the tangential stress in the steady state (arbitrary units), as a function of the inverse wavelength $1 / \Lambda$ (in $\mathrm{cm}^{-1}$ ), and clearly exhibits a rather broad peak, from which we calculate $L_{\text {fluc }} \sim \Lambda \approx 5 \mathrm{~mm}$. For all data, the normal stress is the same, $N \approx 9.73 \mathrm{kPa}$; the power spectrum is obtained for a driving velocity of $880 \mu \mathrm{m} / \mathrm{s}$.

a function of the shearing velocity. Clearly, both quantities are basically independent of the velocity. The tangential stress fluctuations are thus characterized by a typical length scale $L_{\text {fluc }}$, with an order of magnitude of $5 \mathrm{~mm}$. Since the shearing extends on typically ten layers, it means that fluctuations between adjacent layers are of the order of $\pm 500 \mu \mathrm{m}$. A displacement of this size, not very different from a grain radius, is sufficient for a grain to break a contact and create another one, and such phenomena are presumably responsible for the tangential stress fluctuations.

\section{B. Stop-and-go experiments}

In this section, the focus is on the tangential stress and dilatancy response when we stop the external drive and restart it after some waiting time. The first step is to prepare a sample in a steady state. After pouring the grains in the annular cell, we rotate the cell of an interval between two adjacent teeth-that is, a $30^{\circ}$ rotation or a displacement of $5.76 \mathrm{~cm}$ - at a given velocity $V$. Then we proceed to stopand-go experiments, stopping the motor and waiting a given time $t_{\text {wait }}$, from $90 \mathrm{~s}$ up to several hours. During the waiting time, the grains are thus submitted to both normal and tangential stress. Then we restart the motor at the same velocity $V$ which was used to prepare the sample and so on.

A typical result of such an experiment is given in Fig. 5. Let us first focus on the upper curve, which shows the evolution of the (dimensionless) tangential stress. Just after the stop, there is a small decrease in tangential stress and a very slow creep during the waiting time. When restarting the shearing, the interface resists elastically up to a maximum
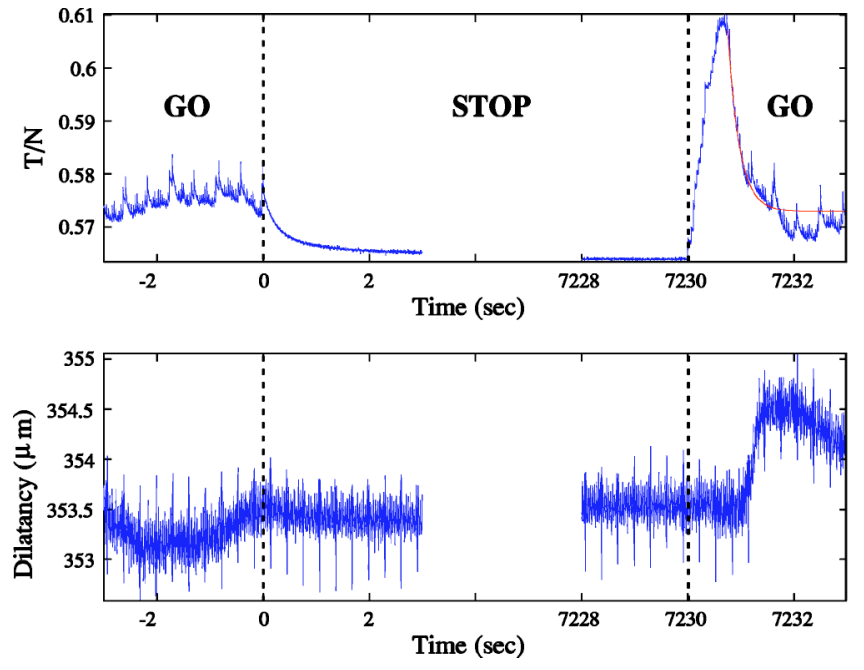

FIG. 5. Evolution of the normalized shear variation (upper curve) and dilatancy variation (lower curve, in $\mu \mathrm{m}$ ) with time, in a stop-and-go experiment. The resolution is $400 \mathrm{point} / \mathrm{s}$ or 9 point $/ \mu \mathrm{m}$. The sample is prepared under a normal stress of $9.73 \mathrm{kPa}$ and a driving velocity of $44 \mu \mathrm{m} / \mathrm{s}$. The dotted lines indicate the stop time $(t \equiv 0 \mathrm{~s})$ and the restart time $(t=7230 \mathrm{~s})$. Here $3 \mathrm{~s}$ are shown just after the stop, and $2 \mathrm{~s}$ just before the restart, during the creep. In the upper curve, we show an exponential fit of the evolution of the tangential stress from its maximum value toward the steady state. The small "spikes" in the tangential stress data may be due to the motor. Their typical frequency is roughly 2 times the number of steps per seconds, and they disappear when the motor is stopped. The high-frequency oscillations, in the dilatancy data, are instrumental noise. They are related to the electronics, not to the motor, since they persist during the stop.

value of the tangential stress. It means that during the stop under normal and tangential stress there has been an evolution of the effective (static) friction coefficient. The system exhibits aging. If we pursue the displacement of the grains, sliding occurs and the tangential stress recovers its steadystate value, at the same level as before the stop. There is rejuvenation of the granular layer under shearing.

Let us define $\Delta \mu$ as the difference between the value of $T / N$ at the peak and its value in the steady state. This quantity is plotted as a function of the waiting time in Fig. 6; to estimate the error made in the measurements, ten identical stop-and-go experiments (same sample, same waiting time of $30 \mathrm{mn}$ ) have been done. The standard deviation of the measurements for the $\Delta \mu$ peak and the relaxation time $\tau$ (see below) give the error bars in Figs. 6 and 7. The quantity $\Delta \mu$ increases roughly as the logarithm of the waiting time, and in all experiments the slope ranges in the interval $[0.01,0.02]$ per unit logarithm. We do not observe any significant evolution of the slope with the imposed driving velocity. This logarithmic increment of the effective static friction coefficient has been observed by de Ryck et al. [5], with a slope of 0.01 , for normal stresses of the same order of magnitude as in our experiment. It is also observed for a quartz gouge between granite blocks at high normal stress (25 MPa) with a slope $0.005[15,16]$. In Fig. 6, we show two sets of data. Open circles correspond to experiments done just after sample preparation (pouring the grains and $30^{\circ}$ rotation at 


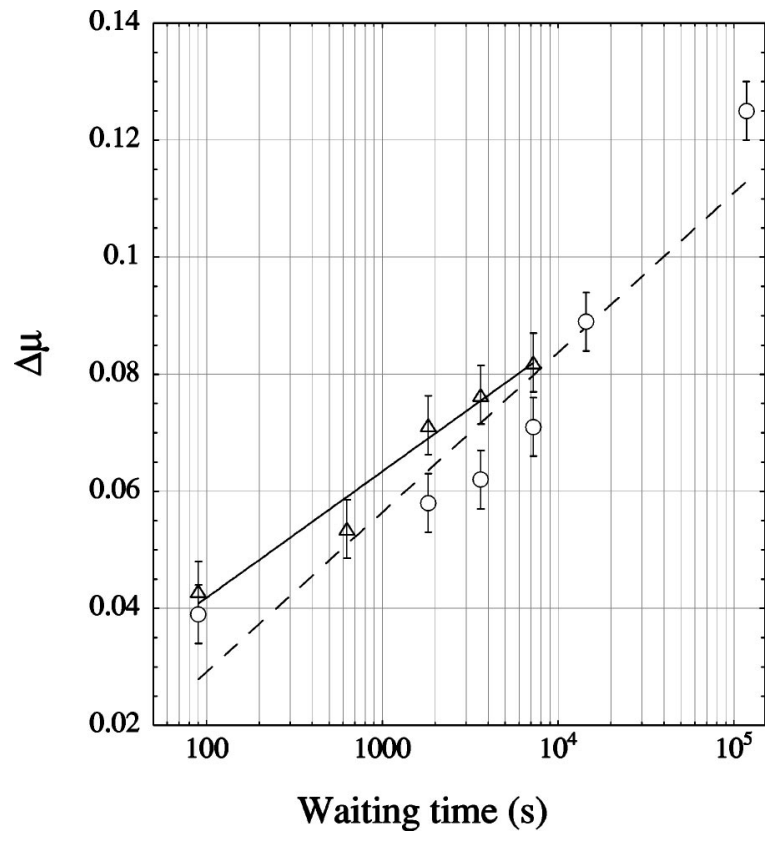

FIG. 6. Plot of the friction coefficient peak $\Delta \mu$ at restarting (linear scale), as a function of the waiting time (logarithmic scale). The two different symbols correspond to the same stop-and-go experiment, done on the same sample, just after sample preparation $(\bigcirc)$ and after the first experiment $(\triangle)$. The slope of the fit, which corresponds to the coefficient $b$ of Eq. (2), is 0.010 in the first case and 0.012 in the second case. The velocity for sample preparation and restart was $22 \mu \mathrm{m} / \mathrm{s}$, and normal stress was $10.78 \mathrm{kPa}$.

constant driving velocity). The logarithmic fit is not good at small waiting time. A curve of the same shape has already been observed by Losert et al. [14] (see their Fig. 5), for a stop-and-go experiment in a linear geometry, at comparable driving velocity $(28.7 \mu \mathrm{m}$ or $0.25 \mathrm{~d} / \mathrm{s}$ in units of their grain diameter) and waiting times, but a very small normal stress of $20 \mathrm{~Pa}$.

This phenomenology is also observed in solid friction experiments $[25,26]$. In this context, the contact of the two rough solids occurs between microasperities, and aging is interpreted as a yielding of those microasperities under stress. This leads to an increase of the actual contact area between the two solids during the waiting time, hence an increase in the friction coefficient. Resuming the shear, sliding begins and the microcontacts are progressively destroyed and replaced by new contacts between fresh asperities. Sliding implies rejuvenation of the frictional interface. The phenomenological rate and state model of Dieterich, Rice, and Ruina [27,28] (DRR) has been introduced to describe the dependence of the friction coefficient $\mu$ on sliding velocity $\dot{x}$ and on an internal variable $\phi$ describing the age of contacts. In this model,

$$
\begin{gathered}
\mu=\mu_{0}+a \ln \frac{\dot{x}}{V_{0}}+b \ln \frac{\phi V_{0}}{D_{c}}, \\
\frac{d \phi}{d t}=1-\frac{\phi \dot{x}}{D_{c}},
\end{gathered}
$$

where $\mu_{0}$ is the steady-state friction coefficient at constant sliding velocity $V_{0}, D_{c}$ is a characteristic length, and $a$ and $b$ two material-dependent constants. Those equations must be completed by one describing the dynamics. Neglecting the inertia, this last equation may be written

$$
K(V-\dot{x})=W \frac{\mathrm{d} \mu}{\mathrm{d} t}
$$

where $K$ is the spring stiffness and $W$ is the weight supported by the grains.
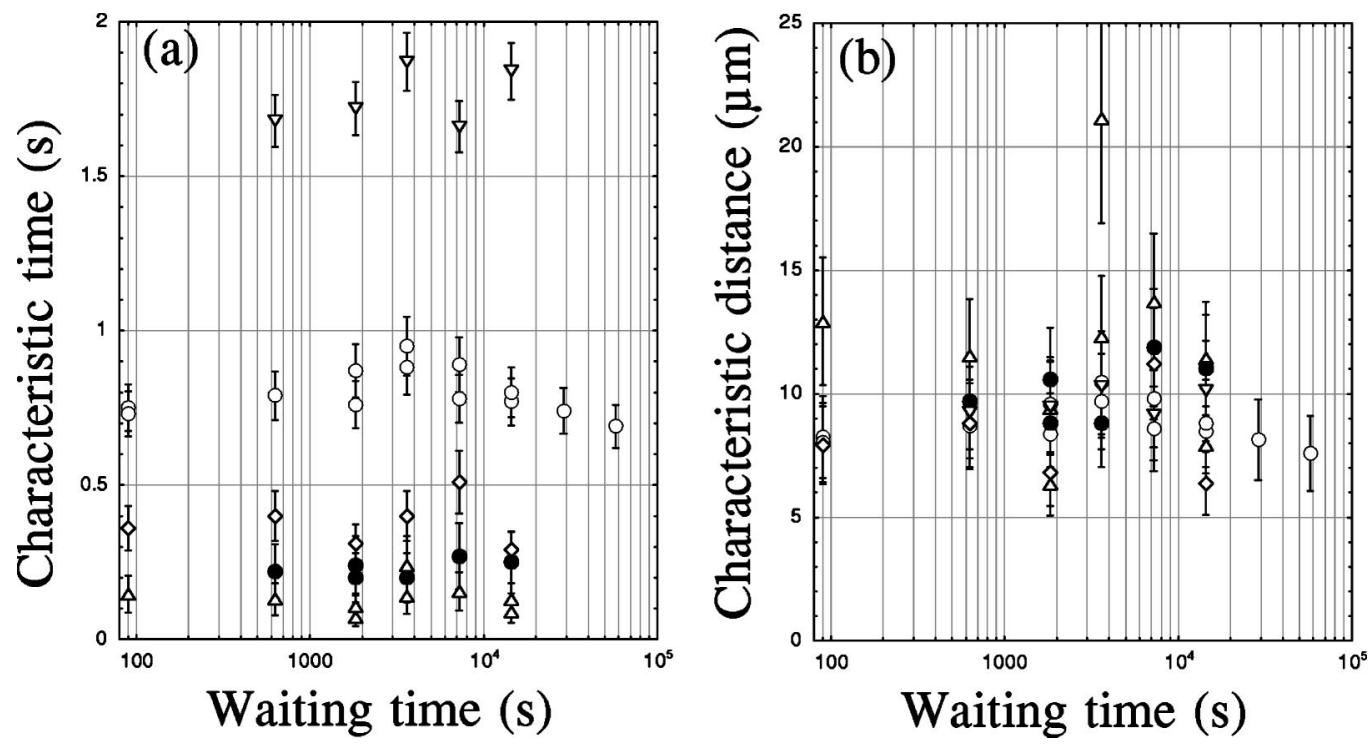

FIG. 7. (a) Plot of the characteristic time $\tau$ as a function of the waiting time, for stop-and-go experiments made at several different driving velocities $V(\nabla, 5.5 \mu \mathrm{m} / \mathrm{s} ; \bigcirc, 11 \mu \mathrm{m} / \mathrm{s} ; \diamond, 22 \mu \mathrm{m} / \mathrm{s} ; \bullet, 44 \mu \mathrm{m} / \mathrm{s} ; \triangle, 88 \mu \mathrm{m} / \mathrm{s})$. (b) Plot of the product $\tau \times V$, as a function of the waiting time, for the same experiments (same symbols). It is clear that the data collapse around a mean value of $10 \mu \mathrm{m}$. 
The state variable $\phi$ is basically the age of the contact. Without sliding-that, is $\dot{x}=0-\phi=t$ and the model includes the logarithmic increase of the static coefficient of friction with contact time. Formally, there is a divergence in Eq. (2) at $\dot{x}=0$ but any measurement of the friction coefficient implies that the surface undergoes slip at some scale [2]. Under constant driving velocity $V$, the steady-state solution of Eqs. (2)-(4) is

$$
\dot{x}=V, \quad \phi^{*}=D_{c} / V, \quad \mu_{d}=\mu_{0}+(a-b) \ln \left(V / V_{0}\right) .
$$

When sliding sets in, the structural age $\phi$ decreases from its initial value, after a waiting time $t_{\text {wait }}$, toward the smaller value $\phi^{*}$ reached in steady motion: the DRR model includes rejuvenation of the interface as well.

The sliding velocity is equal to the driving velocity $V$ in the steady state. It is also the case just at the peak value [26]. Indeed, $K(V-\dot{x})=W \mathrm{~d} \mu / \mathrm{d} t=0$ just at the peak. Hence a rough estimate of the relaxation of $\phi$ is obtained if we assume $\dot{x}$ $=$ const $=V$. A small perturbation $\phi=\phi^{*}+\delta \phi$ evolves following

$$
\frac{d \delta \phi}{d t}=-\frac{V}{D_{c}} \delta \phi,
$$

which corresponds to an exponential relaxation with the characteristic time $\tau \equiv D_{c} / V$. Since $\delta \phi$ is a small perturbation, $\mu$ is linear in $\delta \phi$ and its relaxation toward steady state occurs with the same time scale $\tau$. The length $D_{c}$ is thus identified as the characteristic length necessary for contact rejuvenation to occur. In what follows, we will use those results obtained in a linear geometry, because as we said before the radius of the annular cell is much greater than its width and curvature effects may be neglected [5].

Let us focus now on the relaxation toward the steady state for the tangential stress in our granular system. As shown in Fig. 5, it is quite well described by an exponential fit. In the case of the figure, the characteristic time is $0.27 \mathrm{~s}$. We made a systematic study of similar stop-and-go experiments, varying only the driving velocities. The results are displayed in Fig. 7. From Fig. 7(a), we see that this relaxation time is independent of the waiting time, but depends on the shearing velocity $V$. When we plot the product $V \tau$, all the data collapse on a single curve as shown in Fig. 7(b). This means that the relaxation is described by a characteristic length, the value of which is about $10 \mu \mathrm{m}$. Another way to see this is to plot $\tau$, averaged on all waiting times at a given angular velocity $\Omega$, as a function of $1 / \Omega$. This is done in Fig. 8 .

The order of magnitude for $D_{c}$ is microscopic, but much larger than in the context of solid friction, where $D_{c}$ $\approx 0.5 \mu \mathrm{m}$, an order of magnitude roughly comparable with that of a microcontact between asperities [25]. A length scale much smaller than the bead radius and much greater than the typical size of asperities, which appears naturally in a granular piling, is the size of the contacts between the grains. This latter may be roughly estimated if we consider that the normal stress is borne by "columns" of grains. Let $S$ be the area of the cell and $d$ the mean grain diameter. There are roughly $S / d^{2}$ columns of grains that bear the normal force $N S$, for a normal stress $N$. The force on each column is thus $N d^{2}$. Us-

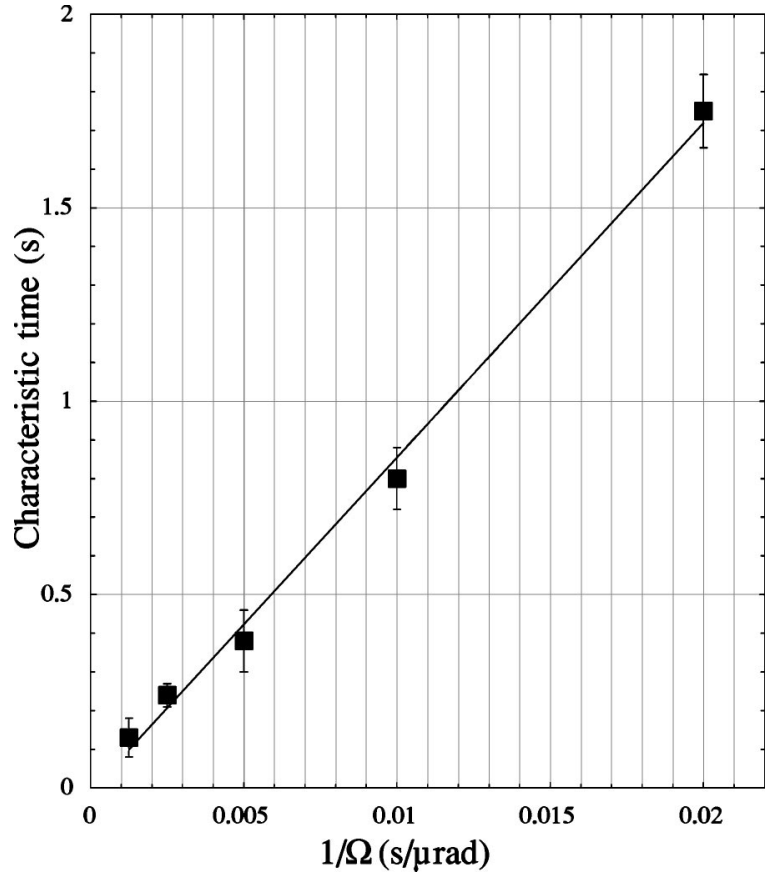

FIG. 8. Plot of the characteristic time taken by the tangential stress to evolve toward its stationary value, averaged over all waiting times, as a function of the inverse angular driving velocity $1 / \Omega$. The error bars are the standard deviation around the mean value.

ing Hertz theory of the contact between spheres ([29], Sec. 9 ), we get for the contact diameter $a$ a value ranging between 8 and $12 \mu \mathrm{m}$, for normal stresses ranging between 3.3 and $11.3 \mathrm{kPa}$. This order of magnitude compares very well with that of $D_{c}$. We think that this comparison is relevant, because a column of grains behaves like a chain of frictional contacts, which before sliding behaves like a chain of springs resisting tangential stress. Sliding will occur at the weaker contact, relaxing the stress in the rest of the chain: the typical length necessary to relax the stress in the chain is thus the size of this weaker contact. This picture is of course oversimplified, because we consider only one column and sliding occurs on all the annulus surface. Nevertheless, we think that it is reasonable to consider that initially the sliding is concentrated on a single contact, rather than being shared among all contacts. We tried to exhibit a variation of $D_{c}$ with the normal stress. It is not completely convincing, because of the rather large scatter in the data [see Fig. 7(b)] : we measure $9 \pm 3 \mu \mathrm{m}$ for $3.3 \mathrm{kPa}$ and $14 \pm 3 \mu \mathrm{m}$ for $11.3 \mathrm{kPa}$. The only thing that can be said with confidence is that it does not contradict our interpretation of $D_{c}$.

Let us finally discuss the evolution of the dilatancy, shown in the lower curve of Fig. 5. If we consider what happens when we restart the driving, it is perfectly clear, and observed in every such experiment, that the dilatancy does not evolve during the elastic reloading of the granular layer. It means that configurational rearrangements of the grains do not occur in the granular layer at a significant level until the beginning of sliding. The dilatancy evolution at the resuming of sliding is very small, even smaller than typical fluctuations in the steady state. Moreover, it does not depend on the waiting time, as shown in Fig. 9. This is another piece of 


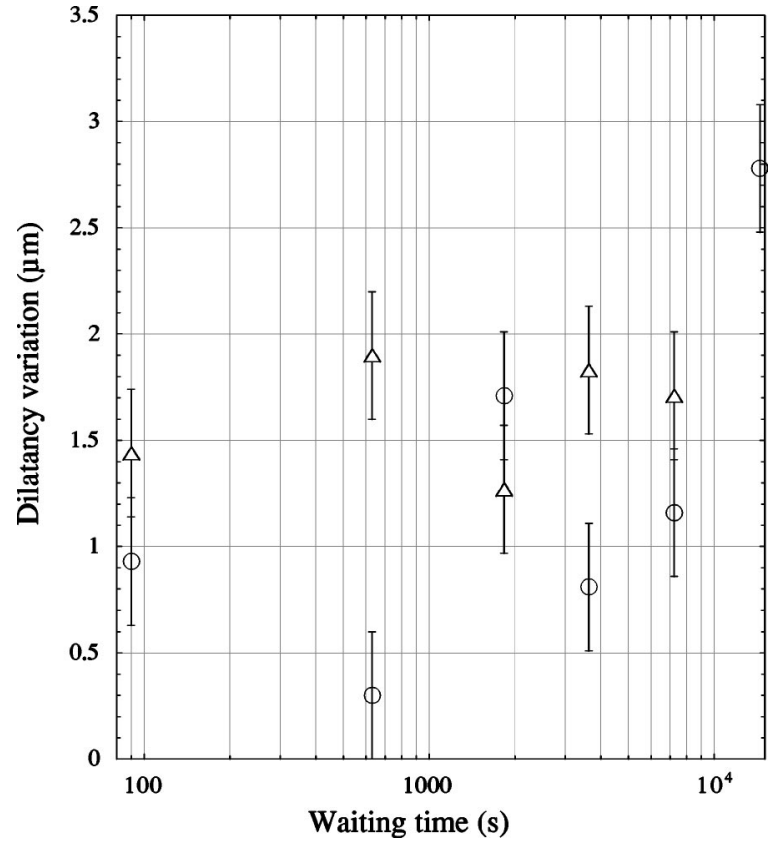

FIG. 9. Plot of the dilatancy variation after restarting as a function of waiting time, in logarithmic scale. The data correspond to the same experiments as in Fig. 6, and the symbols have the same significance. The variation of dilatancy is always very small (compared either to a mean level of $350 \mu \mathrm{m}$ or to fluctuations about $\pm 5 \mu \mathrm{m}$; see Fig. 2) and independent of the waiting time.

evidence that, during the stop, basically no structural rearrangement occurs in the granular layer.

This is consistent with our previous picture of "columns" of grains, in which contacts are established between grains in such a way that they support the weight of the cover. They age during the waiting time, and when we resume the shear they respond elastically, up to a maximum stress. Then sliding occurs, and the dilatancy evolves. This picture is supported by the results of Utter and Behringer et al. [12]. In a 2D Couette experiment at constant volume, with photoelastic grains, they visualize the stress distribution at the grain scale during a stop-and-go experiment. This distribution is not homogeneous, but rather concentrated along stress chains. Those stress chains, established in the steady state, are almost not perturbed during the wait, neither by restarting the shear in the same direction as before the stop. The lack of large grain rearrangements in their observations is consistent with the lack of dilatancy variations during the stop which is seen in our experiments. Aging, as evidenced by the peak in tangential stress at restarting shown in Fig. 5, is thus not explained by structural rearrangements of the piling, but rather by the behavior of contacts between grains.

When the contacts are between plastic materials, such as contacts between microasperities of PMMA blocks [25], or between quartz grains at very high normal stress [3,7], aging may be interpreted as due to yielding at contacts, which increases the actual contact area, hence the strength of the contact. This is difficult to invoke for brittle material such as glass. Aging at the contacts may be due to some contaminants or to condensation of water vapor.

\section{Velocity jumps}

In the Dieterich-Rice-Ruina model (2), the friction coefficient depends on the sliding velocity. A velocity jump from $V_{-}$to $V_{+}$should lead to a variation of the steady state friction coefficient:

$$
\Delta \mu_{\text {stat }}=(a-b) \ln \frac{V_{+}}{V_{-}} .
$$

A sudden change of velocity is not necessary to observe this effect, which expresses the fact that the steady-state friction coefficient depends on the sliding velocity. We may use the data already used for Fig. 4, since they were obtained for several sliding velocities, but only one initial density. In Fig. 11(a), below, we plot the effective friction coefficient, averaged on a large sliding distance (typically $3 \mathrm{~cm}$ ), as a function of the sliding velocity. The data are consistent with the DDR model, but the error bars, which are given by the amplitude of fluctuations displayed in Fig. 4, are quite large. In this respect, velocity jump experiments give better results.

If the velocity jump is instantaneous, there is another effect: The value of $\phi$ just after the jump is unchanged, $\phi$ $=D_{c} / V_{-}$, and there is an instantaneous increment in the friction coefficient

$$
\Delta \mu_{\mathrm{inst}}=a \ln \frac{V_{+}}{V_{-}} .
$$

The relaxation from the peak $\Delta \mu_{\text {inst }}$ toward the steady state at velocity $V_{+}, \Delta \mu_{\text {stat, }}$, takes places on a characteristic distance equal to $D_{c}$ [2].

We thus perform (quasi-)instantaneous velocity jumps, from $V_{-}$to $V_{+}>V_{-}$. The sample is prepared by initial shearing at uniform driving velocity $V_{-}$during a $30^{\circ}$ rotation. Then, we perform series of jumps with the same $V_{-}$, and several values of $V_{+}$, in increasing and then decreasing order (typically from 0.2 up to $1.8 \mathrm{~mm} / \mathrm{s}$ ). We make sure that the large velocity displacement is sufficient to reach a steady state.

A typical experimental result is given in Fig. 10, showing the (dimensionless) tangential stress in the upper curve, the dilatancy in the lower. We do observe the variation $\Delta \mu_{\text {stat }}$ of the stationary values for the effective friction coefficient. The granular layer is velocity strengthening (i.e., the tangential stress increases with the velocity). Our velocity jumps are performed after large cumulated shear displacements, $6 \mathrm{~cm}$ or 40 bead diameter for each data point, and we always do observe velocity strengthening of the granular layer. This was not the case in the experiments of Mair and Marone [7], who observed a transition from a first velocity strengthening regime toward a velocity weakening one, for a displacement of 100 grain diameters. But in the case of their experiments, they begin the velocity jumps before reaching the steady state. Their velocity strengthening regime is a transient, different in nature from the one that we observe.

In Fig. 11(b), we plot the evolution of the variation of friction coefficient with the higher velocity $V_{+}$, for several lower velocities $V_{-}$. The data follow a logarithmic evolution, and when plotted as a function of the ratio $V_{+} / V_{-}$they collapse onto a single curve, in agreement with the DRR model 

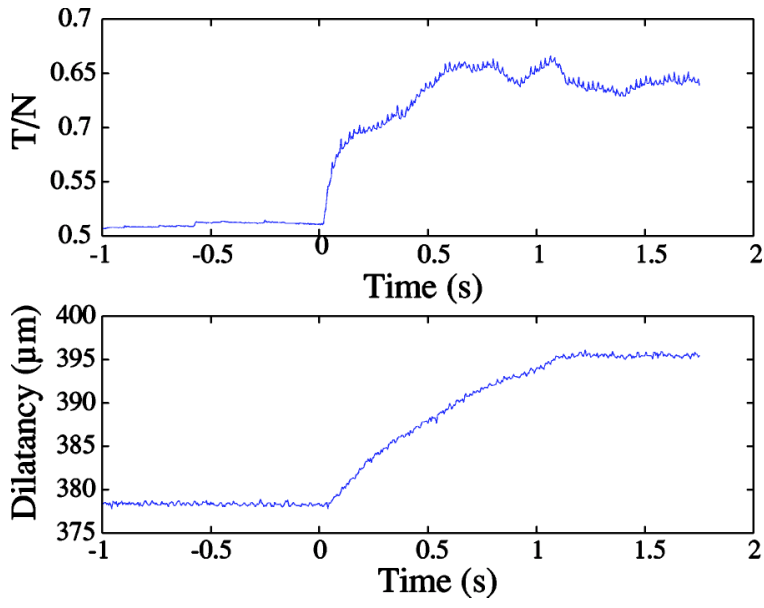

FIG. 10. Example of velocity jump; the upper curve shows the tangential stress, the lower curve the dilatancy, in $\mu \mathrm{m}$, as functions of time, in $\mathrm{s}$. The low velocity is $V_{-}=11 \mu \mathrm{m} / \mathrm{s}(t<0)$, the high velocity is $V_{+}=880 \mu \mathrm{m} / \mathrm{s}(t>0)$. The normal stress is $9.73 \mathrm{kPa}$. The time origin is taken at the jump $V_{-} \rightarrow V_{+}$. The amplitude of dilatancy variation should be compared to the dilatancy fluctuations in steady state, typically $\pm 5 \mu \mathrm{m}$ (see Table I), and to the dilatancy variation in a stop-and-go experiment (see Fig. 9).

(7) [see Fig. 11(c)]. The data are consistent with those of Fig. 11(a), which are obtained in a completely different way. The slope, equal to $a-b$ in the model, is 0.036 and ranges in the interval [ 0.030 .05$]$ in all experiments. With our previous measurement of $b$, this means that $a$ ranges in the interval [0.04 0.07].

We may compare our results to those of the geophysicists [2,3,7], and those of Géminard et al. [8]. Those experiments are quite similar, except for the normal stress which is very low in [8], typically $20 \mathrm{~Pa}$, and very high in [2,3,7], typically $25 \mathrm{MPa}$, to be compared to the order of magnitude of the normal stress in our experiments, $30 \mathrm{kPa}$. Those stresses may be compared directly because the Young's moduli $Y$ of the grains, either glass bead as in the present work ([8], $Y$
$=60 \mathrm{GPa})$ or granite $([2,3,7], Y \in[50,70] \mathrm{GPa})$, are very similar.

No evolution of the friction coefficient is observed in [8], with a velocity varying over four orders of magnitude, from 0.1 up to $1000 \mu \mathrm{m} / \mathrm{s}$, or $10^{-3}$ up to 10 bead diameters per second. Under high stresses, both instantaneous increment $\Delta \mu_{\text {inst }}$ and steady-state increment $\Delta \mu_{\text {stat }}$ are observed in $[2,3,7]$. We do not see the instantaneous increment $\Delta \mu_{\text {inst }}$ in our experiment, in contrast with the DRR model, Eq. (8).

The instantaneous increment $\Delta \mu_{\text {inst }}$ is linked to the state variable $\phi$ that describes aging in Eqs. (2) and (3). Since we do observe aging in our system, it is quite puzzling not to see any instantaneous increment. We will thus discuss at length the possible explanations for our result.

The space resolution of the data is at least $0.11 \mu \mathrm{m}$ between two points, at velocity $V_{+}$. This is much less than the value of $D_{c} \approx 10 \mu \mathrm{m}$, so that a lack of resolution is not a relevant explanation.

The variations of friction coefficient are rather small compared to the fluctuations in the steady state, and we must make large changes in velocity (by a factor of 20, typically). It is thus not obvious that the velocity change is actually instantaneous. The rotation is ensured by a stepping motor, and the maximum angular acceleration is 0 to $0-40^{\circ} / \mathrm{s}$ in $250 \mathrm{~ms}$, hence $\dot{V}_{\max }=0.3 \mathrm{~m} / \mathrm{s}^{2}$. In all cases, the low velocity is negligible. For a high velocity ranging from 0.2 up to $1.8 \mathrm{~mm} / \mathrm{s}$, the duration of the accelerated motion ranges between 0.36 and $5.7 \mathrm{~ms}$, corresponding to a distance between 0.02 and $5.1 \mu \mathrm{m}$. Even in the worst case, this distance is less than $D_{c}$ (for the case of Fig. 10, the motion is accelerated for the first $1.25 \mu \mathrm{m}$, much less than $D_{c}$ ). Hence it does not seem that an insufficient acceleration should be the explanation.

Assuming that the instantaneous change of driving velocity creates a sudden jump in friction, this implies further deformation of the cantilever spring, hence the motion of the cover plate. The typical time scale for cover motion is $\sqrt{m /(2 k)}$, where the factor of 2 comes from the moment of
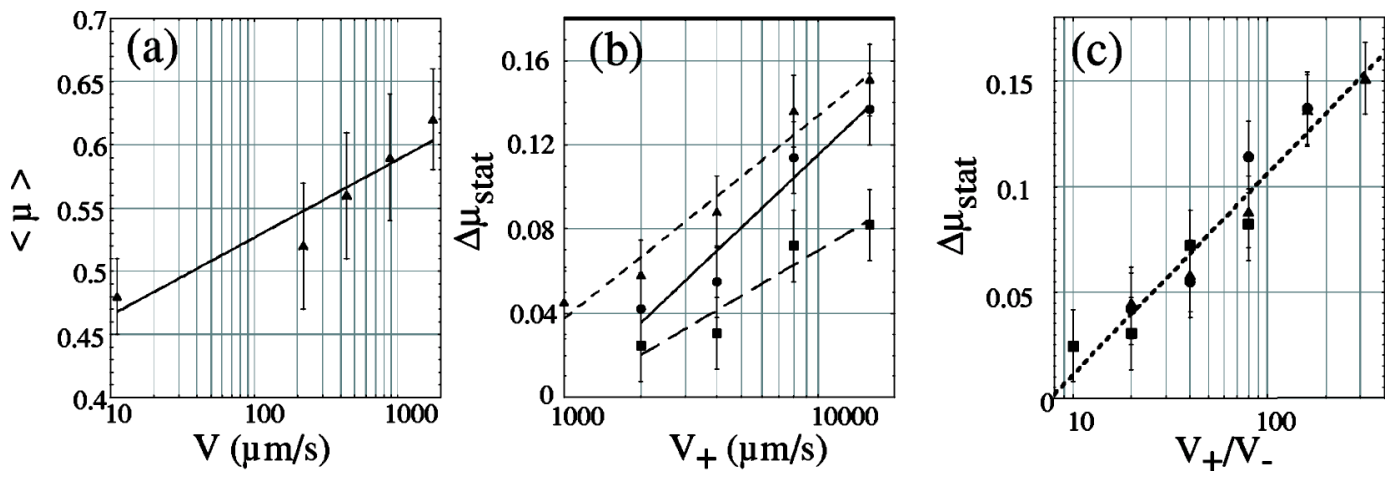

FIG. 11. (a) Value of the effective friction coefficient $\langle\mu\rangle$ (averaged over a large distance, typically $3 \mathrm{~cm}$ ), in the steady state, as a function of the velocity $V$ in a logarithmic scale. Data are the same as those used in Fig. 4. Note that error bars (typically \pm 0.05 ) are much lager than in the case of (b) (typically \pm 0.02 ), due to the measuring process. (b) Value of the increment of effective friction coefficient $\Delta \mu_{\text {stat }}$ for a velocity jump $V_{-} \rightarrow V_{+}$, in the steady state, as a funtion of $V_{+}$. The abcissa is given in a logarithmic scale, for several values of $V_{-}$: $V_{-}=5.5 \mu \mathrm{m} / \mathrm{s}$ (solid triangles), $V_{-}=11 \mu \mathrm{m} / \mathrm{s}$ (soild circles), and $V_{-}=22 \mu \mathrm{m} / \mathrm{s}$ (solid squares). (c) Same data, with the same symbols, but plotted as functions of the ratio $V_{+} / V_{-}$, in logarithmic scale. All data collapse on a single curve. The normal stress is $9.73 \mathrm{kPa}$. The error bars are estimated from the fluctuations of $T / N$. For (a), those fluctuations are displayed in Fig. 4. For (b) and (c) they are much less since they are calculated on a much shorter distance. 
inertia of a plain disk of mass $m$. In the case of Fig. 10, $m$ $=9.6 \mathrm{~kg}$ and this time scale is only 2.5 times less than $D_{c} / V$; it is not obvious to consider the cover motion as instantaneous, so that we cannot exclude completely inertial effects.

The physical reason for the instantaneous jump $\Delta \mu_{\text {inst }}$, in the context of solid friction, is that the system has to slide on a distance $D_{c}$ for the complete renewal of asperities [27]. In experiments on granular gouge [2,3,7], the thinest granular particles created by wear of the rocks surface and comminution of the initial grain layer should play the role of the asperities. Such phenomena are not relevant for our experiments, which are done at much smaller normal stresses. Another point is that, in order to see aging in our system, we have to wait typically hundreds of seconds: no effect is seen for a waiting time of 1 or $10 \mathrm{~s}$. The steady state value $\phi^{*}$, for a sliding at the lowest velocity of $5 \mu \mathrm{m} / \mathrm{s}$, is $2 \mathrm{~s}$, which is probably not enough to see any effect.

A careful measurement of the coefficient $a$ of Eq. (2) has been made by de Ryck et al. [5], and they found good agreement between the model and their data. They used an annular shearing box, normal loads comparable to ours, and siliga gel grains of typical size $0.1 \mathrm{~mm}$. But they measured $a$ during the creep in a stop-and-go experiment, not by imposing velocity jumps on the grains. In the creep phase, the response of the granular layer is mainly due to the behavior of the frictional contacts, without significant rearrangements of grains, which is definitely not the case for a velocity jump.

As shown by Fig. 10, an increment of dilatancy follows a driving velocity jump. Such a dilatancy increment has been observed by Mair and Marone [7], but not by Géminard et al. [8]. It is very difficult to compare those results, because of the very different orders of magnitude for the normal stresses. In the experiments of Mair and Marone [7], the grains are progressively broken and the granularity is not constant during the shearing, which is not the case in our experiment. They nevertheless measure a dilatancy increment of typically $2 \%$ of the mean grain diameter, for $V_{+} / V_{-}=10$ and a layer of 40 grains. This is roughly the same in our experiments (see Fig. 12; the increment is $10 \mu \mathrm{m}$ for $V_{+} / V_{-}=20$ and a layer of 15 grains). The lack of dilatancy increment when varying the driving velocity, evidenced in the experiments of Géminard et al. [8], is probably due to the very low normal stress in their experiments. In fact, they always observe a smaller dilatancy than we do. When they shear freshly poured grains at constant driving velocity, the dilatancy increment is $5 \mu \mathrm{m}$ or $d / 20$, for a layer of depth $30 d$, in units of the beads diameter $d$. In the same kind of experiment, we obtain a dilatancy of $d / 3$ for a layer of $15 d$.

The dilatancy increment depends on the amplitude of the driving velocity jump. The evolution of the dilatancy is shown in Fig. 12, as a function of $V_{+} / V_{-}$. It evolves logarithmically, with slope $13 \mu \mathrm{m}$ per unit of the quantity $\ln \left(V_{+} / V_{-}\right)$. The dilatancy variation (typically $50 \mu \mathrm{m}$ ) is significantly greater than the dilatancy fluctuations in steady state $( \pm 5 \mu \mathrm{m})$. However, this variation is quite small when compared to the dilatancy of freshly poured grains (typically $500 \mu \mathrm{m})$. For $V_{+} / V_{-} \approx 100$, the dilatancy increment is about $50 \mu \mathrm{m}$. Dividing by the number $(\approx 10)$ of granular layers below the teeth extremities, we get $5 \mu \mathrm{m}$, which is comparable to the roughness of the beads. Thus the mecanism of

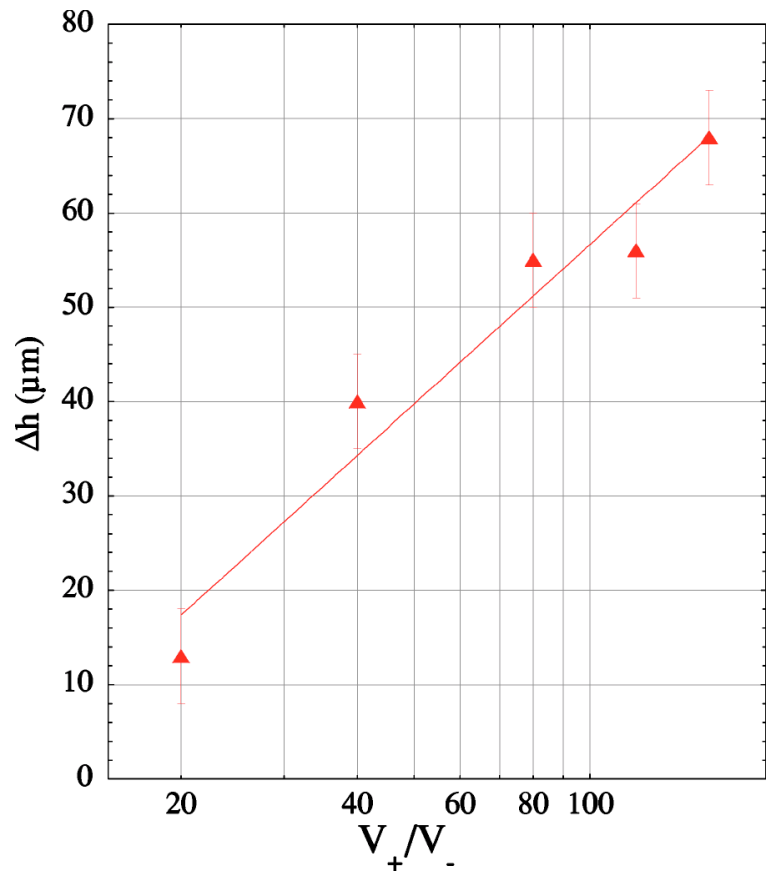

FIG. 12. Plot of the increment of dilatancy, in $\mu \mathrm{m}$, for a velocity jump $V_{-} \rightarrow V_{+}$, as a function of the ratio $V_{+} / V_{-}$in logarithmic scale. The normal stress is $9.73 \mathrm{kPa}$.

the dilatancy variation during a velocity jump may be related to the behavior of individual frictional contacts, whereas the shearing of freshly poured grains necessarily involves structural rearangements of the grains.

\section{CONCLUSION}

We have studied the low-velocity shearing of a confined granular layer. The dimensionless shear rate varies between $10^{-6}$ and $10^{-4}$ [Eq. (1)].

A first result is the evidence that the response of the granular layer under shearing is described by characteristic length scales, not time scales. Those length scales describe the evolution of the layer toward a steady state and the stress fluctuations when this steady state is reached. Importantly, the steady state for tangential stress is reached on the same length scale as that for dilatancy. It shows that there is an intricate coupling between the tangential stress variations and those of dilatancy, or free volume.

When the driving is stopped and the grains are kept under normal and tangential stress, the system ages, showing a logarithmic increase of static friction coefficient with waiting time. There is no evolution of the dilatancy during either the stop or elastic reloading of the granular layer at the begining of restart. Aging is thus not due to structural rearrangements of the granular layer, which would imply changes in density. The contacts between the grains, established at the stop and supporting the stress during the waiting time, are responsible for aging.

Resuming the shearing, there is rejuvenation of the system. Several experiments, done at different driving velocities, provide evidence that this rejuvenation occurs with a 
characteristic length that may be identified with the parameter $D_{c}$ of the Dieterich-Rice-Ruina model. We find $D_{c}$ $\approx 10 \mu \mathrm{m}$, which is the order of magnitude of a Hertz contact between adjacent grains. The cover plate, during the waiting time, is held by several chains of contacting grains. Each contact resists to shear up to a maximum value, and the chains are broken when the weakest contact breaks. A length scale of the order of the lateral size of a Hertz contact is thus sufficient for this process to take place everywhere in the cell, such that sliding occurs and rejuvenates the granular layer.

When driving velocity jumps are imposed on the grains, the system exhibits velocity strengthening behavior. The variation of friction coefficient, in the steady state, is a logarithmic function of the upper velocity $V_{+}$. Data obtained for several lower-velocity $V_{-}$collapse onto a single curve when plotted as a function of $\ln \left(V_{+} / V_{-}\right)$. No instantaneous increase is observed for the friction coefficient. This cannot be related to a poor resolution, neither to insufficient acceleration.
When the higher velocity is kept constant on a sufficient distance, a steady state is reached for the dilatancy, and the dilatancy increase is a logarithmic function of $V_{+} / V_{-}$. The order of magnitude of this effect indicates that the response of the layer probably does not involve structural rearrangements of the grains.

With our experimental protocol, the stop-and-go and velocity jump experiments are done when the shear band is fully established. In this regime, our measurements are well described by the Dieterich-Rice-Ruina model [21,22], which does not include the dilatancy, which thus appears as a slaved variable. On the contrary, the dynamics of shear band building, just after filling the cell, exhibits a strong coupling between tangential stress and dilatancy.

\section{ACKNOWLEDGMENTS}

I thank A. de Ryck and A. Lemaître for useful discussions. I am indebted to T. Baumberger and C. Caroli for a critical reading of the manuscript and many useful remarks.
[1] R. M. Nedderman, Statics and Kinematics of Granular Materials (Cambridge University Press, Cambridge, England, 1992).

[2] C. Marone, Annu. Rev. Earth Planet Sci. 26, 643 (1998).

[3] N. M. Beeler, T. E. Tullis, M. L. Blanpied, and J. D. Weeks, J. Geophys. Res. 101, 8697 (1996).

[4] M. Lubert and A. de Ryck, Phys. Rev. E 63, 021502 (2001).

[5] A. de Ryck, R. Condotta, and M. Lubert, Eur. Phys. J. E 11, 159 (2003).

[6] S. Shibuya, T. Mitachi, and S. Tamate, Geotechnique 47, 769 (1997).

[7] K. Mair and C. Marone, J. Geophys. Res. 104, 28899 (1999).

[8] J.-C. Géminard, W. Losert, and J. P. Gollub, Phys. Rev. E 59, 5881 (1999).

[9] J.-C. Tsai, G. A. Voth, and J. P. Gollub, Phys. Rev. Lett. 91, 064301 (2003).

[10] G. Chambon, J. Schmittbuhl, A. Corfdir, J. P. Vilotte, and S. Roux, Phys. Rev. E 68, 011304 (2003).

[11] D. Fenistein and M. Van Hecke, Nature (London) 425, 256 (2003).

[12] B. Utter and R. P. Behringer, Eur. Phys. J. E 14, 373 (2004).

[13] R. R. Hartley and R. P. Behringer, Nature (London) 421, 928 (2003).

[14] W. Losert, J.-C. Géminard, S. Nasuno, and J. P. Gollub, Phys. Rev. E 61, 4060 (2000).
[15] S. L. Karner and C. Marone, Geophys. Res. Lett. 25, 4561 (1998).

[16] N. M. Beeler and T. E. Tullis, J. Geophys. Res. 102, 22595 (1997).

[17] S. Torquato, T. M. Truskett, and P. G. Debenedetti, Phys. Rev. Lett. 84, 2064 (2000).

[18] J. Duran, Sables, Poudres et Grains (Eyrolles, Paris, 1997).

[19] GDR MiDi Collaboration, Eur. Phys. J. E 14, 341 (2004).

[20] S. Douady and A. Daerr, in Physics of Dry Granular Media, edited by H. J. Herrmann, J.-P. Hovi, and S. Luding (Kluwer, Dordrecht, 1998).

[21] A. Lemaître, Phys. Rev. Lett. 89, 064303 (2002).

[22] A. Lemaître, Phys. Rev. Lett. 89, 195503 (2002).

[23] J. Desrues, R. Chambon, M. Mokni, and F. Mazerolle, Geotechnique 46, 529 (1996).

[24] M. Oda and H. Kazama, Geotechnique 48, 465 (1998).

[25] T. Baumberger, P. Berthoud, and C. Caroli, Phys. Rev. B 60, 3928 (1999).

[26] L. Bureau, T. Baumberger, and C. Caroli, Eur. Phys. J. E 8, 331 (2002).

[27] J. Dieterich, J. Geophys. Res. 84, 2161 (1979).

[28] J. R. Rice and A. L. Ruina, J. Appl. Mech. 50, 343 (1983).

[29] L. D. Landau and E. M. Lifshitz, Theory of Elasticity (Pergamon Press, Oxford, 1986). 